\title{
Non-fermentative gram-negative bloodstream infection in northern Italy: a multicenter cohort study
}

\author{
Renato Pascale ${ }^{1 *} \mathbb{D}$, Silvia Corcione ${ }^{2}$, Linda Bussini ${ }^{1}$, Livia Pancaldi ${ }^{1}$, Daniele Roberto Giacobbe ${ }^{3,4}$, \\ Simone Ambretti ${ }^{5}$, Tommaso Lupia ${ }^{2}$, Cristina Costa ${ }^{6}$, Anna Marchese ${ }^{7,8}$, Francesco Giuseppe De Rosa ${ }^{2}$, \\ Matteo Bassetti ${ }^{3}$, Claudio Viscoli ${ }^{3}$, Michele Bartoletti ${ }^{1}$, Maddalena Giannella ${ }^{1}$ and Pierluigi Viale ${ }^{1}$
}

\begin{abstract}
Background: The management of non-fermentative gram-negative bloodstream infection (NFGN-BSI) offers numerous challenges. In this study the aim is to analyse a large cohort of patients with NFGN-BSI recruited in the northern Italy to describe epidemiology, etiological and susceptibility pattern, therapeutic management and outcome.

Methods: Multicentre retrospective cohort study of patients hospitalised at three large teaching hospitals in northern Italy in a fourth year period.

Results: 355 BSI episodes were analyzed, due to P. aeruginosa (72.7\%), A. baumannii (16.6\%), and Stenotrophomonas maltophilia (10.7\%). Overall, 21.4\% of isolates were defined as DTR, highest rate among A. baumannii (64.4\%). All-cause 30-day mortality rate was $17.5 \%$. Rates of XDR or DTR A. baumannii isolation were significantly higher in non-surviving patients. Independent risk factors for 30-day mortality were: age (HR 1.03, 95\%Cl 1.00-1.04, $p=0.003)$, septic shock ( HR 2.84, 95\%Cl 1.67-4.82, p<0.001) and BSI due to Acinetobacter baumannii (HR 2.23, 95\%Cl 1.27-3.94, p=0.005).
\end{abstract}

Conclusion: The overall prevalence of DTR was high in the NFGN BSI cohort analyzied, mainly among Acinetobacter baumannii episodes (64.4\%). Acinetobacter baumannii is showed to be an independent predictor of mortality. These evidences marked the urgent need of new therapeutic options against this pathogen.

Trial registration number: 79/2017/O/OssN. Approved: March14th, 2017.

Keywords: Bloodstream infection, Non-fermentative gram-negative bacteria, Multidrug resistance, Difficult to treat resistance, Therapeutic management, 30-Day mortality

\section{Background}

The management of non-fermentative gram-negative bloodstream infection (NFGN-BSI) offers numerous challenges. Indeed, there are several clinical and microbiological issues that may contribute to its high morbidity

*Correspondence: renato.pascale@aosp.bo.it

1 Infectious Diseases Unit, Department of Medical and Surgical Sciences, IRCCS Azienda Ospedaliero-Universitaria di Bologna, Via Massarenti 11, 40137 Bologna, Italy

Full list of author information is available at the end of the article and mortality. NFGN-BSI is usually diagnosed in people with severe underlying conditions, critically ill and/or immunocompromised patients [1-3]. Isolates are generally resistant, or prone to acquire resistance, to first-line antibiotics resulting in a high rate of initial inappropriate therapy and/or in the use of less effective and more toxic drugs. To counteract these findings, antibiotic combination regimens are frequently employed with controversial results in terms of efficacy, toxicity and collateral environmental damage $[3,4]$. 
The knowledge of local epidemiology, etiological distribution in terms of causative agents and antibiotic resistance, therapeutic approach and factors associated with poor outcome are useful to guide infection control and antimicrobial stewardship policies and to inform clinicians regarding the best treatment approach [4].

With this premise, we analysed a large cohort of patients with NFGN-BSI recruited in three regions of northern Italy to describe the current epidemiology, etiological and susceptibility pattern distribution, therapeutic management and outcome.

\section{Methods}

\section{Study design and setting}

Multicentre retrospective cohort study of patients hospitalised at three large teaching hospitals in northern Italy: (i) Sant'Orsola Malpighi Hospital, Bologna; (ii) City of Health and Sciences, Molinette Hospital, Turin; (iii) San Martino Hospital, Genoa. The study period was from January 1st 2013 to December 31st 2016. Patients were identified through the records of the Microbiology Laboratory of each hospital.

Data source was the clinical charts and hospital records, reviewed until 90 days after the index blood cultures (BCs). Study variables were collected using a case report form. Data accuracy was assessed by a senior investigator. In addition, the numbers of patient days per year were recorded to assess the incidence of NFGN-BSI in the participating hospitals during the study period.

The study was approved by the institutional Ethics Committee of coordinating center, Sant'Orsola Malpighi Hospital (Comitato Etico di Area Vasta Emilia Centro-CE-AVEC79/2017/O/OssN). According to local rules, due to the retrospective nature of the study, the acquisition of consent by the interested parties was not envisaged in case of organizational impossibility. The collection of informed consent was obtained in all cases in which it was possible to provide adequate information to the patients. Patients were aware that samples could be used in research and that data could be published. Data were collected anonymously.

The antibiotic treatment, both empirical and definitive, was selected according to clinical judgment and not dictated by study protocol. As for polymyxin based therapy, colistin is the only drug available in Italy.

\section{Participants}

All adult ( $\geq 18$ years) patients diagnosed with NFGNBSI were included in the study. NFGN-BSI was defined as one or more positive BCs obtained from a patient suspected of having infection. Patients were considered only once at the time of first episode (index BCs).
Exclusion criteria included: (i) polymicrobial BSI, defined as growth of more than one micro-organism, excluding potential contaminants (i.e. coagulase-negative staphylococci, Corynebacterium spp., Propionibacterium spp.); (ii) clinical data not available.

\section{Variables and definitions}

The primary outcome was all-cause mortality within 30 days after index BCs [5]. The predictor variables included age and sex. The Charlson comorbidity index was used to asses underlying disease [6]. Immunosuppression included: neutropenia (neutrophil count $<500$ / $\mathrm{mm}^{3}$ ), solid organ transplantation, hematopoietic stem cell transplantation, corticosteroid therapy (at a dosage higher then or equivalent to prednisone $16 \mathrm{mg} /$ day $\geq 15$ days), uncontrolled human immunodeficiency virus (HIV) infection $\left(<200 \mathrm{CD} 4 / \mathrm{mm}^{3}\right)$.

BSI was classified into nosocomial, healthcare-associated and community acquired using Friedman's criteria [7]. Clinical severity at infection onset was assessed according to Sequential Organ Failure Assessment (SOFA) score and septic shock criteria [8]. BSI sources were established according to Centers for Disease Control and Prevention (CDC) criteria [9]. BSI was considered as primary in case of unrecognized source. BSI was defined as complicated when the infection source was not fully removable.

The susceptibility pattern of isolates was classified according to Magiorakos et al. criteria [10] as multidrug resistance (MDR) as nonsusceptibility to $\geq 1$ agent in $\geq 3$ antimicrobial categories; extensive drug resistance (XDR) as susceptibility limited to $\leq 2$ categories; and pan-drug resistance (PDR) as nonsusceptibility to all agents in all antimicrobial categories.

In addition, CDC surveillance definitions were used to assess susceptibility to carbapenems, extended-spectrum cephalosporins (ESC) and fluoroquinolones (FQ) (https://gis.cdc.gov/grasp/PSA/Downloads/AR-Pheno typeDefinitions.pdf). Moreover susceptibility to betalactam/betalactamase inhibitors (BL/BLI) and colistin was determined according to European Committee for Antimicrobial Susceptibility Testing (EUCAST) criteria.

The new definition of "difficult to treat resistance" (DTR) was also assessed as reported elsewhere [11, 12]. Empirical therapy was defined as antibiotics administered before the susceptibility report was available. Appropriate empirical therapy was defined as a therapy contained at least one in vitro active drug (according to the susceptibility pattern of the isolate) administered within $24 \mathrm{~h}$ after drawing index BCs. Inappropriate empirical therapy was defined as inactive antibiotic administration or a delayed antibiotic therapy. Antibiotic treatment administered according to susceptibility results was considered as 
definitive antibiotic therapy. Antibiotic regimens including more than one anti-gram-negative agents, irrespective of their in vitro activity against the BSI isolate, during more than $50 \%$ of treatment duration were defined as "combination regimen". Antibiotic therapy including at least two drugs showing in vitro activity against the BSI isolate was labelled as " 2 -in vitro active combination regimen". Duration of antibiotic treatment was defined as the number of consecutive days during which the patient received an appropriate antibiotic regimen. Source control was defined as the removal of the infection source within 7 days of index BCs, including the performance of non-surgical or surgical procedures to treat an obstructive focus or abscess at any site including, among others, the urinary tract, biliary tract and surgical site, and the removal of any device deemed as the source of BSI.

\section{Microbiology}

BCs were incubated using the BACTEC FX Automated Blood Culture System (Becton Dickinson, Franklin Lakes, NJ). All positive BCs were processed with Maldi Biotyper MALDI-TOF system (Bruker Daltonics, Bremen, Germany) for rapid and reliable species identification of microorganisms. Antimicrobial susceptibility testing was performed using the Vitek 2 automated system (bioMerieux, Marcy l'Etoile, France) in two hospitals (Bologna and Genova) and the MicroScan system in the remaining hospital (Torino). The minimum inhibitory concentrations (MICs) were interpreted using EUCAST clinical breakpoints for all tested antibiotics.

\section{Statistical analysis}

For the descriptive analysis, categorical variables were presented as absolute numbers and their relative frequencies. Continuous variables were presented as the mean and standard deviation if normally distributed or as the median and interquartile range (IQR) if non-normally distributed.

Univariate and multivariate analysis were used to assess risk factors for all-cause 30-day mortality. Categorical variables were compared using $\chi^{2}$ or Fisher exact test when appropriate. Continuous variables were compared using the Mann-Whitney U-test. Significant and clinically relevant covariates identified in univariate analysis were introduced into a multivariable Cox regression survival model after verifying for proportional hazards and collinearity. Significance was considered for $\mathrm{p}<0.05$. All the analysis were performed using SPSS software.

\section{Results}

Over the study period, 527 patients were diagnosed with NFGN-BSI. Of them, 172 were excluded: 146 patients had polymicrobial bacteremia, and for the
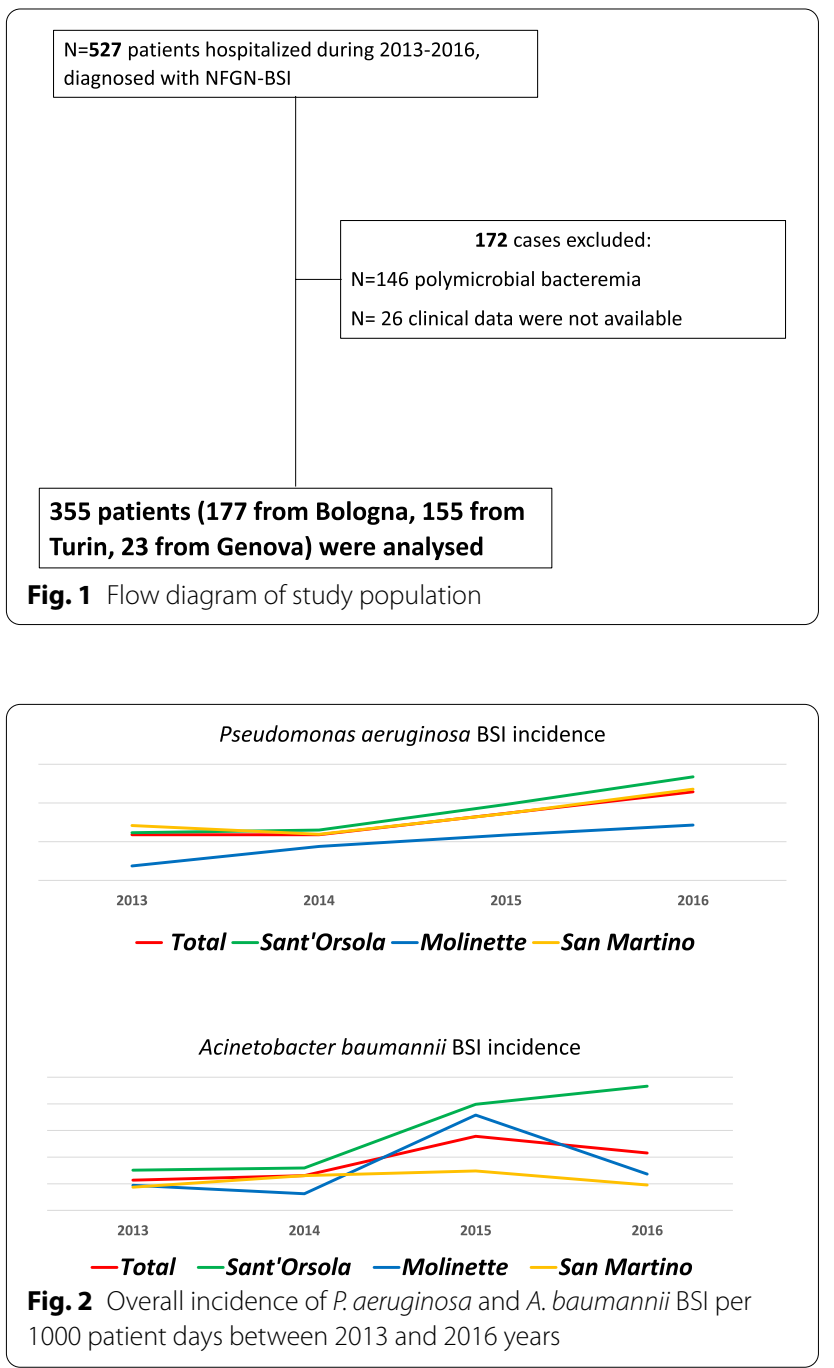

remaining 26 patients clinical data were not available. Thus, 355 patients (177 from Bologna, 155 from Turin and 23 from Genova) were analysed (Fig. 1).

The overall incidence of Pseudomonas aeruginosa BSI per 1000 patient days was $0.12,0.12,0.17$, and 0.23 in 2013, 2014, 2015 and 2016, respectively. It was similar between participating hospitals. The overall incidence of Acinetobacter baumannii BSI per 1000 patient days was $0.02,0.03,0.06$, and 0.04 in 2013, 2014, 2015 and 2016, respectively. It was similar between participating hospitals. Data shown in Fig. 2.

Etiological distribution and susceptibility patterns are shown in Table 1. Most episodes were due to $P$. aeruginosa $(\mathrm{n}=258,72.7 \%)$, followed by $A$. baumannii $(\mathrm{n}=59,16.6 \%)$, and Stenotrophomonas maltophilia $(\mathrm{n}=38,10.7 \%)$. Overall, $21.4 \%$ of isolates were defined as DTR with highest rate among $A$. baumannii (64.4\%). Susceptibility rates to individual antibiotic categories 
Table. 1 Causative agents and their susceptibility patterns of monomicrobial NFGN-BSI during 2013-2016 in three tertiary teaching hospitals from northern Italy

\begin{tabular}{lllllll}
\hline & N & MDR & XDR & PDR & CR & DTR \\
\hline Pseudomonas aeruginosa & 258 & $15(5.8)$ & $53(20.5)$ & $/$ & $82(31.8)$ & $35(13.6)$ \\
Acinetobacterbaumannii & 59 & 0 & $37(62.7)$ & $1(1.7)$ & $41(69.7)$ & $38(64.4)$ \\
Stenotrophomonas maltophilia & 38 & $/$ & $/$ & $/$ & $/$ & $2(5.3)$ \\
\hline
\end{tabular}

Resistance categories (MDR, XDR, PDR) were mutually exclusive while antibiotic class resistances (CR) and new definition (DTR) were not

$C R$ carbapenem resistance, $D T R$ difficult-to-treat resistance, $M D R$ multidrug-resistance, $P D R$ pandrug-resistance, $X D R$ extensively drug-resistance

are shown in Fig. 3. Both $P$. aeruginosa and A. baumannii maintained high rate susceptibility to colistin.

The general characteristics of study population are shown in Table 2 . Overall, $65.6 \%$ of patients were male, the median age was 67 (IQR 55-79) years, the median Charlson index was 5.7 (IQR 3.6-7.4), and 24.5\% were immunocompromised. Most patients were hospitalized at a medical ward at BSI onset (59.2\%), and the majority of episodes were hospital acquired (70.1\%). Infection source was not identified in $183(51.5 \%)$ cases, in the remaining cases the most common sources of NFGN-BSI were CVC $(n=102)$ and lower respiratory tract $(n=49)$.

As shown in Fig. 4, data on empirical and definitive antibiotic therapy were available for 266 (75\%) and 333 (94\%) patients, respectively. Active therapy was administered in $35.2 \%$ and $73.8 \%$ of empiric and definitive cohort patients, respectively. Empiric combination regimens were used in $55(15.5 \%)$ patients with a 2 -in vitro active drugs in 16 (4.5\%). Combination regimens were used in $32.4 \%$ of patients in the definitive cohort, $16.3 \%$ with 2 -in vitro active drugs. Empiric and definitive antibiotic regimens according to isolates are shown in Additional file 1: Tables S1 and S2.

All-cause 30 -day mortality rate was $17.5 \%$. Relapse at 90 days was observed in 7 patients (2\%) within a median of 30 days (IQR 17-50) after index BCs. Compared to patients who were alive at day 30 (Table 2), non-surviving patients exhibited higher age, Charlson index and SOFA score, and higher rate of septic shock. Rates of $A$. baumannii, XDR or DTR isolation were significantly higher in non-surviving patients, while the rates of $P$. aeruginosa isolation and empirical active therapy were higher among survivors. For definitive therapy, the use of any combination was associated with higher mortality while that of 2-in vitro active combination did not. At multivariate analysis adjusted for DTR, Stenotrophomonas maltophilia BSI, Acinetobacter baumanni BSI, Pseudomonas aeruginosa BSI, active empiric therapy, active targeted therapy and source control, the independent risk factors for all-cause 30-day mortality were: age (odds ratio, OR 1.03, 95\%, confidence interval, CI 1.00-1.04, $\mathrm{p}=0.003$ ), septic shock (OR 2.84, 95\%CI 1.67-4.82, $\mathrm{p}<0.001$ ) and BSI due to Acinetobacter baumannii (OR 2.23, 95\%CI $1.27-3.94, \mathrm{p}=0.005$ ). Furthermore, risk factors for 30-day mortality have been estimated separately among patients with $P$. aeruginosa (Additional file 1: Table 3), $A$. baumannii (Additional file 1: Table 4) and S. maltophilia BSI (Additional file 1: Table 5).

\section{Discussion}

In our large cohort of NFGN-BSI we have found high rates of DTR and carbapenem resistance, especially among A. baumannii. Empirical active therapy administration was significantly higher in surviving patients at univariate analysis, however it was not confirmed at multivariate analysis. Combination therapy, also with 2 active

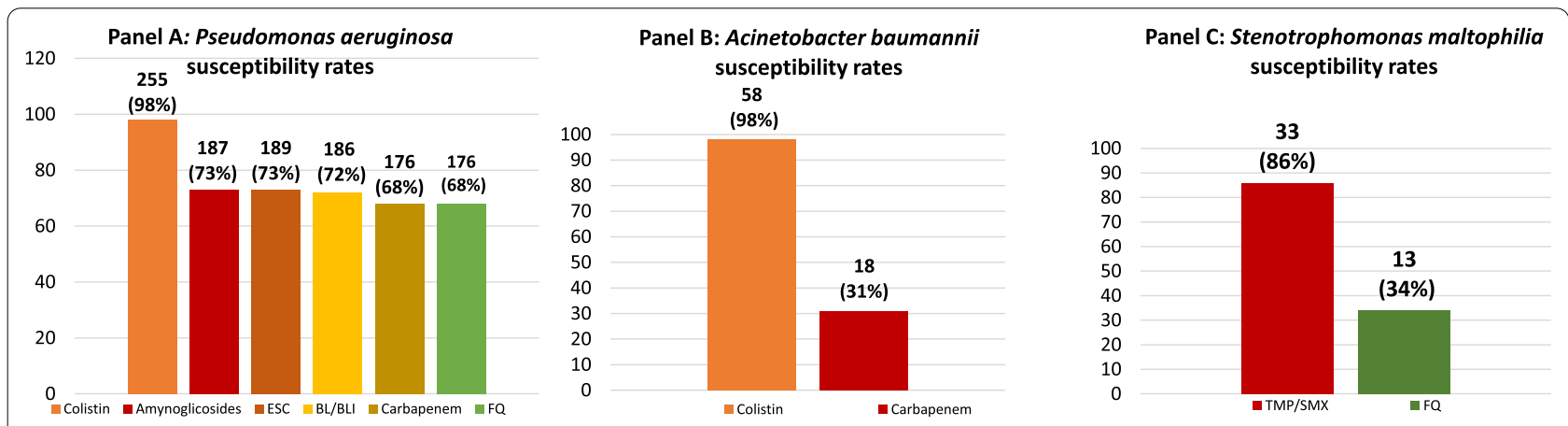

Fig. 3 Susceptibility rates to individual antibiotic categories for Pseudomonas aeruginosa, Acinetobacter baumannii and Stenotrophomonas malptophilia. BL/BL/ betalactam/betalactamase inhibitor, ESC extended-spectrum cephalosporin, FQ fluoroquinolone resistance 
Table. 2 Univariate analysis of risk factors for all-cause 30-day mortality

\begin{tabular}{|c|c|c|c|c|}
\hline & TotaIN = $355(\%)$ & SurvivorsN = $293(\%)$ & Non-survivorsN = 62 (\%) & $p$ \\
\hline \multicolumn{5}{|l|}{ Demographics } \\
\hline Age (years) (median, IQR) & $67(55-79)$ & $67(54-78)$ & $73(61-84)$ & 0.016 \\
\hline Male sex & $233(65.6)$ & $194(66.2)$ & $39(62.9)$ & 0.66 \\
\hline \multicolumn{5}{|l|}{ Comorbidities } \\
\hline Charlson index (median, IQR) & $5.7(3.6-7.4)$ & $5.2(3.5-7.1)$ & $6.25(4-9)$ & 0.034 \\
\hline Immunosuppression & $87(24.5)$ & $74(25.3)$ & $13(21)$ & 0.52 \\
\hline Ward of admission & & & & 0.22 \\
\hline Medical & $210(59.2)$ & $176(60.1)$ & $34(54.8)$ & \\
\hline ICU & $80(22.5)$ & $61(20.8)$ & 19 (30.6) & \\
\hline Surgical & $65(18.3)$ & $56(19.1)$ & $9(14.5)$ & \\
\hline Site of BSI acquisition & & & & 0.078 \\
\hline Community acquired & $71(20.0)$ & $65(22.2)$ & $6(9.7)$ & \\
\hline Healthcare associated & $35(9.9)$ & $27(9.2)$ & $8(12.9)$ & \\
\hline Hospital acquired & $249(70.1)$ & $201(68.6)$ & $48(77.4)$ & \\
\hline CRE carrier at BSI onset & $44(12.1)$ & $35(11.9)$ & $9(14.5)$ & 0.462 \\
\hline \multicolumn{5}{|l|}{ Clinical severity at BSI onset } \\
\hline SOFA (median, IQR) & $3(2-5)$ & $3(2-5)$ & $4(3-6)$ & 0.005 \\
\hline Septic shock & $59(16.6)$ & $38(13)$ & $21(33.9)$ & 0.001 \\
\hline \multicolumn{5}{|l|}{ Source of BSI } \\
\hline Undefined & $183(51.5)$ & $150(51.2)$ & $33(53.2)$ & 0.782 \\
\hline CVC related & $102(28.7)$ & $86(29.4)$ & $16(25.8)$ & 0.645 \\
\hline Lower respiratory tract & 49 (13.8) & $39(13.3)$ & $10(16.1)$ & 0.686 \\
\hline Biliary tract & $41(11.5)$ & $34(11.6)$ & $7(11.3)$ & 1 \\
\hline Urinary tract & $31(8.7)$ & $27(9.2)$ & $4(6.5)$ & 0.624 \\
\hline Intra-abdominal & $18(5.1)$ & $16(5.5)$ & $2(3.2)$ & 0.551 \\
\hline Complicated BSI & $38(10.7)$ & $27(12.9)$ & $11(21.6)$ & 0.125 \\
\hline Etiology & & & & 0.009 \\
\hline Pseudomonas aeruginosa & $258(72.7)$ & $222(75.8)$ & $36(58.1)$ & 0.005 \\
\hline Acinetobacter baumannii & $59(16.6)$ & $41(14)$ & $18(29)$ & 0.005 \\
\hline Stenotrophomonas maltophilia & $38(10.7)$ & $30(10.2)$ & $8(12.9)$ & 0.651 \\
\hline Resistance phenotypes* & & & & 0.005 \\
\hline MDR & $34(9.6)$ & $22(8.4)$ & $12(22.2)$ & \\
\hline$X D R$ & $58(16.3)$ & $46(17.5)$ & $12(22.2)$ & \\
\hline \multicolumn{5}{|l|}{ Antibiotic class resistance* } \\
\hline ECR & $95(26.7)$ & $77(26.3)$ & $18(29)$ & 0.752 \\
\hline$B L / B L I R$ & $78(22)$ & $55(18.8)$ & $23(37.1)$ & 0.002 \\
\hline$C R$ & $124(34.9)$ & $93(31.7)$ & $31(50)$ & 0.008 \\
\hline $\mathrm{FQR}$ & $144(40.6)$ & $107(36.5)$ & $37(59.7)$ & 0.001 \\
\hline AminoglycosidesR & 98 (27.6) & $69(26.4)$ & $29(51.8)$ & $<0.001$ \\
\hline TMP/SMXR & $53(14.9)$ & $39(21.9)$ & $14(29.2)$ & 0.337 \\
\hline COLIR & $5(1.4)$ & $5(1.7)$ & $0(0)$ & 0.592 \\
\hline \multicolumn{5}{|l|}{ New definition* } \\
\hline DTR & $75(21.1)$ & $52(17.7)$ & $23(37.1)$ & 0.001 \\
\hline \multicolumn{5}{|l|}{ Therapeutic management } \\
\hline ID consultation & $148(41.7)$ & $119(40.6)$ & $29(46.8)$ & 0.397 \\
\hline Source control & $131(36.9)$ & $111(37.9)$ & $20(32.3)$ & 0.470 \\
\hline Appropriate empirical therapy & $125(35.2)$ & $111(38.4)$ & $14(23.7)$ & 0.037 \\
\hline Combination empirical therapy & $55(15.5)$ & $42(18.9)$ & $13(29.5)$ & 0.152 \\
\hline 2 In vitro active combination empirical therapy & $16(4.5)$ & $16(12.8)$ & 0 & 0.223 \\
\hline
\end{tabular}


Table. 2 (continued)

\begin{tabular}{|c|c|c|c|c|}
\hline & TotalN = $355(\%)$ & SurvivorsN = $293(\%)$ & Non-survivorsN $=62(\%)$ & $p$ \\
\hline Appropriate definitive therapy & $262(73.8)$ & $222(76.8)$ & $40(67.8)$ & 0.184 \\
\hline Combination definitive therapy & $115(32.4)$ & $89(32)$ & $26(47.3)$ & 0.031 \\
\hline $\begin{array}{l}2 \text { In vitro active combination definitive therapy } \\
\text { (with drugs) }\end{array}$ & $58(16.3)$ & $48(20.7)$ & $10(23.8)$ & 0.682 \\
\hline
\end{tabular}

$B L / B L I R$ betalactam/betalactamase inhibitor resistance, $B S /$ bloodstream infection, COLIR colistin resistance, $C R$ carbapenem resistance, $C R E$ carbapenem-resistant Enterobacteriaceae, CVC central venous catheter, DTR difficult-to-treat resistance, $E C R$ extended-spectrum cephalosporin resistance, FQR fluoroquinolone resistance, ICU intensive care unit, IQR interquartile range, ID Consultation Infectious Disease Consultation, MDR multidrug-resistance, SOFA sequential organ failure assessment, $T M P / S M X R$ trimethoprim/sulfamethoxazole resistance, XDR extensively drug-resistance

* Resistance categories were mutually exclusive while antibiotic class resistances and new definition (DTR) were not

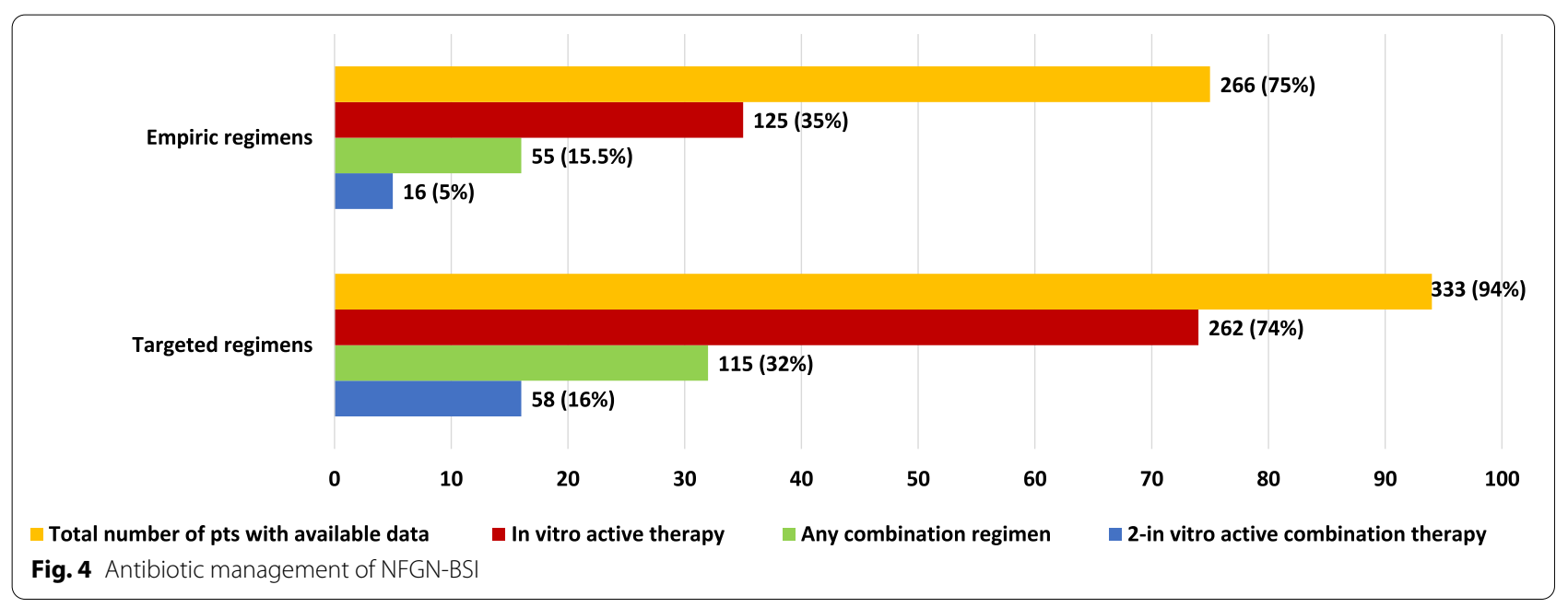

drug, was not associated to improving surviving at multivariate analysis. Of note, $A$. baumannii isolation resulted as independent risk factors for mortality at multivariate analysis. In our cohort nosocomial infections accounted for a large majority of cases (70\%), mainly central venous catheter (CVC) related and pneumonia, according to literature $[13,14]$. Therefore, promote and improve infection control programs would play a critical role in reducing the rates of this kind of nosocomial infections.

The increasing importance of the NFGN bacteria is also related to their complex antimicrobial resistance profile. In our cohort carbapenem resistance showed high prevalence, with $31.8 \%$ and $64.4 \%$ rates for P. aeruginosa and $A$. baumannii respectively. We have also analysed the prevalence of the new definition DTR. This definition reflects the use of second-line agents with poorer therapeutic index, resulting in a better prediction of poor outcome. In our cohort, the overall prevalence of DTR was $21.1 \%$. It varied across species being highest among $A$. baumannii BSI with rates of $64.4 \%$. This value was much higher than data present in literature $[11,15]$. Similarly, DTR prevalence for P.aeruginosa BSI, accounted for $13.6 \%$, higher than the rates showed previously $[11,15]$. As expected, in $A$. baumannii strain, CR and DTR rates were comparable.

In our study active therapy did not result statistically associated with improved outcome as previously reported elsewhere. This finding deserves further investigation. Indeed, the classical way to define if an antimicrobial agent is useful to treat an infection is the MIC determination of strains. However, MIC determination have some concerns: (i) clinical laboratories cannot determine MICs with sufficient accuracy owing to the assay variation in the MIC test especially when automated or semi-automated methods are used, (ii) the MIC does not represent a concentration directly compared with in vivo concentration found during treatment; (iii) bacterial growth conditions in vitro could be different from those in vivo [16]. Also, the in vitro activity of antimicrobial often does not reflect the clinical feasibility due to the specific pharmacokinetic/ toxicodynamic profile of the drugs and the source of infection [17]. These considerations could explain why in our cohort active therapy seems not associated to 
improving surviving. Similar experiences were previously reported elsewhere [18].

In our study, A. baumannii was an independent predictor of mortality. This is line with the characteristics of this pathogen that is commonly responsible for severe opportunistic nosocomial infections mainly in hospitalized immunocompromised patients [1-3]. Additionally, the complex antimicrobial resistance profile and the limited therapeutic arsenal for this strain may explain this result. In this scenario, polymyxins remains in vitro the most active agent. However, the in vitro activity of polymyxins not reflect the clinical feasibility due to the suboptimal pharmacokinetic/toxicodynamic profile of this class [17]. In all the study centers the polymyxin used for the treatment of $A$. baumannii BSI was colistin.

Our study has a number of limitations. Although we have analysed a large cohort of patients in three different centres, the results could be influenced by the epidemiology of a restricted area of our country. Also, our cohort is from all large tertiary teaching hospital reflecting the complexity and epidemiology of patients managed in similar institutions. The retrospective collection of patient and microbiological data could have limited integrity and accuracy. However, a senior investigator and three young investigators revised all clinical report forms (CRFs), and reconciled data reports and missing data with the medical records before including information in the database.

\section{Conclusions}

To conclude, the overall prevalence of DTR was high in our NFGN BSI cohort, mainly among Acinetobacter baumannii episodes. Furthermore, Acinetobacter baumannii is showed to be an independent predictor of mortality. These evidences marked the urgent need of new therapeutic options against this pathogen.

\begin{abstract}
Abbreviations
BCs: Blood cultures; CDC: Centers for Disease Control and Prevention; CR: Carbapenem resistance; CRFs: Clinical report forms; CVC: Central venous catheter; DTR: Difficult to treat resistance; ESC: Extended-spectrum cephalosporins; EUCAST: Eeuropean Committee for Antimicrobial Susceptibility Testing; FQ: Fluoroquinolones; HIV: Human immunodeficiency virus; OR: Odds ratio; $\mathrm{Cl}$ : Confidence interval; IQR: Interquartile range; MDR: Multi drug-resistant; MIC: Minimal inhibitory concentration; NFGN-BSI: Non-fermentative gram-negative bloodstream infection; PDR: Pan drug-resistant; SOFA score: Sequential organ failure assessment score; XDR: Extensively drug-resistant.
\end{abstract}

\section{Supplementary Information}

The online version contains supplementary material available at https://doi. org/10.1186/s12879-021-06496-8.

Additional file 1: Table S1. Empiric therapy according to isolates. Table S2. Definitive therapy according to isolates. Table S3. Univariable and multivariable analysis of risk factors for all-cause 30 -day mortality in patients with Pseudomonas aeruginosa BSI. Table S4. Univariable and multivariable analysis of risk factors for all-cause 30-day mortality in patients with Acinetobacter baumannii BSI. Table S5. Univariable analysis of risk factors for all-cause 30-day mortality in patients with Stenotrophomonas maltophilia BSI*.

\section{Acknowledgements}

Not applicable.

\section{Authors' contributions}

RP: data analysis and drafting the manuscript. SC: data collection. LB: data collection. LP: data collection. DRG: data collection. SA: support in data collection. TL: data collection. CC: support in data collection. AM: support in data collection. CV: support in study design. FGDR: support in study design. MB: manuscript revision. MG: study design, data analysis and manuscript revision. PV: study design and manuscript revision. All authors read and approved the final manuscript.

\section{Funding}

No external funding was received for the present study.

\section{Availability of data and materials}

The original data and materials from this study are available from the corresponding author on reasonable request.

\section{Declarations}

\section{Ethics approval and consent to participate}

The study was approved by the institutional Ethics Committee of coordinating center, Sant'Orsola Malpighi Hospital (Comitato Etico di Area Vasta Emilia Centro-CE-AVEC79/2017/O/OssN). The study was approved in March14th, 2017. This study used data collected from patient records while maintaining patient anonymity. According to local rules, the acquisition of consent by the interested parties was not envisaged in case of organizational impossibility. The collection of informed consent was obtained in all cases in which it was possible to provide adequate information to the patients.

\section{Consent for publication}

Patients enrolled were aware that samples could be used in research and that data could be published and informed consent was obtained. Data were collected anonymously.

\section{Competing interests}

All authors no reported competing interests.

\section{Author details}

${ }^{1}$ Infectious Diseases Unit, Department of Medical and Surgical Sciences, IRCCS Azienda Ospedaliero-Universitaria di Bologna, Via Massarenti 11, 40137 Bologna, Italy. ${ }^{2}$ Department of Medical Sciences, Infectious Diseases, University of Turin, Turin, Italy. Infectious Diseases Unit, Ospedale Policlinico San Martino-IRCCS, Genoa, Italy. ${ }^{4}$ Department of Health Sciences (DISSAL), University of Genoa, Genoa, Italy. ${ }^{5}$ Operative Unit of Clinical Microbiology, Policlinico Sant'Orsola Malpighi, University of Bologna, Bologna, Italy. ${ }^{6}$ Department of Public Health and Pediatrics, Laboratory of Microbiology and Virology, Città della Salute e della Scienza Hospital, University of Turin, Turin, Italy. ${ }^{7}$ Department of Surgical Sciences and Integrated Diagnostics (DISC), University of Genoa, Genoa, Italy. ${ }^{8}$ Microbiology Unit, Ospedale Policlinico San Martino-IRCCS, Genoa, Italy.

Received: 5 August 2020 Accepted: 29 July 2021

Published online: 12 August 2021

\section{References}

1. Mesaros N, Nordmann P, Plesiat P, Roussel-Delvallez M, Van Eldere J, Glupczynski Y, et al. Pseudomonas aeruginosa: resistance and therapeutic 
options at the turn of the new millennium. Clin Microbiol Infect. 2007;13:560-78.

2. Perez F, Hujer AM, Hujer KM, Decker BK, Rather PN, Bonomo RA. Global challenge of multidrug-resistant Acinetobacter baumannii. Antimicrob Agents Chemother. 2007;51:3471-84.

3. Paterson DL. The epidemiological profile of infections with multidrugresistant Pseudomonas aeruginosa and Acinetobacter species. Clin Infect Dis. 2006;43(Suppl 2):S43-48.

4. Gniadek TJ, Carroll KC, Simner PJ. Carbapenem-resistant non-glucosefermenting gram-negative bacilli: the missing piece to the puzzle. J Clin Microbiol. 2016;54:1700-10.

5. Harris PNA, McNamara JF, Lye DC, Davis JS, Bernard L, Cheng AC, et al. Proposed primary endpoints for use in clinical trials that compare treatment options for bloodstream infection in adults: a consensus definition. Clin Microbiol Infect. 2017;23:533-41.

6. Charlson ME, Pompei P, Ales KL, MacKenzie CR. A new method of classifying prognostic comorbidity in longitudinal studies: development and validation. J Chronic Dis. 1987;40:373-83.

7. Friedman ND, Kaye KS, Stout JE, McGarry SA, Trivette SL, Briggs JP, et al. Health care-associated bloodstream infections in adults: a reason to change the accepted definition of community-acquired infections. Ann Intern Med. 2002;137:791-7.

8. Singer M, Deutschman CS, Seymour CW, Shankar-Hari M, Annane D, Bauer $M$, et al. The third international consensus definitions for sepsis and septic shock (Sepsis-3). JAMA. 2016;315:801-10.

9. Horan TC, Andrus M, Dudeck MA. CDC/NHSN surveillance definition of health care-associated infection and criteria for specific types of infections in the acute care setting. Am J Infect Control. 2008;36:309-32.

10. Magiorakos AP, Srinivasan A, Carey RB, Carmeli Y, Falagas ME, Giske CG, et al. Multidrug-resistant, extensively drug-resistant and pandrugresistant bacteria: an international expert proposal for interim standard definitions for acquired resistance. Clin Microbiol Infect. 2012;18:268-81.

11. Kadri SS, Adjemian J, Lai YL, Spaulding AB, Ricotta E, Prevots DR, et al. Difficult-to-treat resistance in gram-negative bacteremia at 173 US hospitals: retrospective cohort analysis of prevalence, predictors, and outcome of resistance to all first-line agents. Clin Infect Dis. 2018;67:1803-14.

12. Giannella M, Bussini L, Pascale R, Bartoletti M, Malagrino M, Pancaldi L, et al. Prognostic utility of the new definition of difficult-to-treat resistance among patients with gram-negative bloodstream infections. Open Forum Infect Dis. 2019;6:ofz505.

13. Peleg AY, Seifert $H$, Paterson DL. Acinetobacter baumannii: emergence of a successful pathogen. Clin Microbiol Rev. 2008;21:538-82.

14. McCann E, Srinivasan A, DeRyke CA, Ye G, DePestel DD, Murray J, et al. Carbapenem-nonsusceptible gram-negative pathogens in ICU and nonICU settings in US hospitals in 2017: a multicenter study. Open Forum Infect Dis. 2018;5:241.

15. Huh K, Chung DR, Ha YE, Ko JH, Kim SH, Kim MJ, et al. Impact of difficultto-treat resistance in gram-negative bacteremia on mortality: retrospective analysis of nationwide surveillance data. Clin Infect Dis. 2020. https:// doi.org/10.1093/cid/ciaa084.

16. Mouton JW, Muller AE, Canton R, Giske CG, Kahlmeter G, Turnidge J. MIC-based dose adjustment: facts and fables. J Antimicrob Chemother. 2018;73:564-8

17. Pea F, Viale P. The antimicrobial therapy puzzle: could pharmacokineticpharmacodynamic relationships be helpful in addressing the issue of appropriate pneumonia treatment in critically ill patients? Clin Infect Dis. 2006;42:1764-71.

18. Zak-Doron Y, Dishon Benattar Y, Pfeffer I, Daikos GL, Skiada A, Antoniadou $A$, et al. The association between empirical antibiotic treatment and mortality in severe infections caused by carbapenem-resistant gram-negative bacteria: a prospective study. Clin Infect Dis. 2018;67:1815-23.

\section{Publisher's Note}

Springer Nature remains neutral with regard to jurisdictional claims in published maps and institutional affiliations.
Ready to submit your research? Choose BMC and benefit from:

- fast, convenient online submission

- thorough peer review by experienced researchers in your field

- rapid publication on acceptance

- support for research data, including large and complex data types

- gold Open Access which fosters wider collaboration and increased citations

- maximum visibility for your research: over $100 \mathrm{M}$ website views per year

At BMC, research is always in progress.

Learn more biomedcentral.com/submissions 